\title{
Occurrence and Identification of Pathogenic Vibrio Contaminants in Common Seafood Available in a Chinese Traditional Market in Qingdao, Shandong Province
}

\author{
Xinjie Song ${ }^{1}$, Jinlin Zang ${ }^{2}$, Weisen $\mathrm{Yu}^{3}$, Xuexiang Shi ${ }^{3 *}$ and Yongning $W u^{4}$ \\ ${ }^{1}$ School of Biological and Chemical Engineering, Zhejiang University of Science and Technology, Hangzhou, China, \\ ${ }^{2}$ Qingdao Municipal Hospital, Qingdao, China, ${ }^{3}$ Department of Nutrition and Food Hygiene, Qingdao Center for Disease \\ Control and Prevention, Qingdao Institute of Preventive Medicine, Qingdao, China, ${ }^{4}$ NHC Key Laboratory of Food Safety \\ Risk Assessment, Chinese Academy of Medical Sciences Research Unit (2019RU014), China National Center for Food \\ Safety Risk Assessment, Beijing, China
}

The investigation of the causative agents for foodborne diseases and subsequent development of preventive steps to control the outbreak and related economic loss is the basic goal and priority of a rational food safety program. The entero-pathogenic Vibrio spp., which are Gram negative bacteria inhabiting estuarine ecosystems, are the major

OPEN ACCESS

Edited by: Giovanna Suzzi, University of Teramo, Italy

Reviewed by:

Learn-Han Lee,

Monash University Malaysia, Malaysia

Rosanna Tofalo,

University of Teramo, Italy

*Correspondence:

Xuexiang Shi

shixx2009@163.com

Specialty section: This article was submitted to

Food Microbiology, a section of the journa Frontiers in Microbiology

Received: 21 February 2020 Accepted: 08 June 2020 Published: 30 June 2020

Citation:

Song $X$, Zang J, Yu W, Shi X and

Wu Y (2020) Occurrence and

Identification of Pathogenic Vibrio

Contaminants in Common Seafood

Available in a Chinese Traditional

Market in Qingdao, Shandong

Province. Front. Microbiol. 11:1488.

doi: 10.3389/fmicb.2020.01488 cause of foodborne illness associated with the consumption of raw or undercooked contaminated seafood or shellfish. To survey the Vibrio contamination in sea snails (Neptunea cumingi Crosse and Busycon canaliculatu), a total of 20 samples were collected from traditional market, at Qingdao city in Shandong province, China and analyzed for Vibrio species contamination. Presumptive-positive colonies grown on a specific Vibrio agar-based medium were picked and identified by the VITEK ${ }^{\mathrm{TM}}$. Vibrio alginolyticus, $V$. parahaemolyticus, and $V$. vulgaris were isolated and identified in 11, 8 , and 2 seafood samples, respectively. Among the 8 isolates of $V$. parahaemolyticus. The $V$. parahaemolyticus isolates were further tested for the $t d h$, trh, and th virulence factors. All the $V$. parahaemolyticus isolates were th-positive, however, all of them were tdh-negative. Interestingly $2 V$. parahaemolyticus isolates were positive for trh virulence factor. These results indicated that there is a high incidence of $V$. alginolyticus and $V$. parahaemolyticus in sea snails. Therefore, food safety regulations for fishery auction markets should be established to control these species in addition to other Vibrio pathogenic contaminants. Our study provides the first evidence for the prevalence of Vibrio spp. in sea snail samples from traditional market in the Qingdao province of China.

Keywords: Foodborne disease surveillance, Vibrio alginolyticus, V. parahaemolyticus, V. vulgaris, sea snails

\section{INTRODUCTION}

Sea snail is a nutritious food that contains various desirable nutritional components of a healthy diet. Nevertheless, there are many health risks associated with the careless consumption of seafood products (Zhang et al., 2018). One of the major risks, seen in a few Asian countries, including China, Korea, and Japan, involves the consumption of raw or undercooked seafood 
that may be naturally contaminated by foodborne pathogens present in the marine environment (Weissfeld, 2014). The risk of marine contamination can be increased if the food is mishandled during production and processing, allowing microorganisms to multiply exponentially in favorable environments (Amagliani et al., 2012). Vibrio spp. are Gram negative, facultatively anaerobic, rod shaped, non-spore-forming bacteria that are ubiquitously and naturally present in estuarine waters throughout the world's aquatic environments and are especially resistant to high salt concentrations (Su and Liu, 2007). Vibrio was first identified as a foodborne pathogen in Japan in the 1950s (Fujino et al., 1953; Letchumanan et al., 2019). By the early 1970s, Vibrio parahaemolyticus was recognized as a cause of diarrheal disease worldwide, most commonly in Asia and the United States. Vibrio parahaemolyticus associated with sea foods is one of the leading agent for food borne illness in China and across the world (Chen et al., 2017; Yang et al., 2017). Although cooking destroys this organism, sea snails are sometime eaten raw and will lead to infections associated with $V$. parahaemolyticus. Therefore, factors contributing to Vibrio contamination, including improperly handled seafood products, could pose a risk of exposure to these infectious agents that are transmissible to humans. Several species of the genus Vibrio are associated with foodborne spoilage and contamination of seafood products (Heng et al., 2017; Lee et al., 2018). The route of Vibrio entry into the biliary system remains unknown, and it was assumed to be via cutaneous lesions or due to gastrointestinal translocation upon consumption of contaminated seafood, as in other Vibrio infections (Liu et al., 2011). A few other species are more hazardous to humans, including $V$. parahaemolyticus and Vibrio cholerae, which are known to cause severe intestinal diseases (Kass and Riemann, 2006). The majority of $V$. parahaemolyticus isolates are avirulent, but they are still the leading cause of gastroenteritis linked to seafood consumption in several countries, including the United States (Iwamoto et al., 2010). Some Vibrio spp. pose a significant health risk to the immunocompromised elderly people and children. Vibrio may enter human hosts via wounds or direct consumption of raw seafood, including shellfish (primarily oysters), and frequently lead to septicemia and, ultimately, death in susceptible individuals (Harwood et al., 2004).

Researchers have shown a significant association between rising seawater temperatures and an increase in the number of Vibrio infections, suggesting that global warming might also be a factor affecting the increasing incidents of Vibrio contamination in seafood (Huehn et al., 2014). Over the past few years, $V$. parahaemolyticus strains have often been isolated from fish, shrimp, and other types of seafood. Additionally, Cho et al. (2016) and Mala et al. (2016) isolated V. parahaemolyticus in ready-to-eat food. In the Zhejiang province of China alone, there were 71 outbreaks caused by $V$. parahaemolyticus, resulting in 933 illnesses and 117 hospitalizations from 2010 to 2014. Recently, a multistate outbreak of gastrointestinal illnesses linked to oysters imported from Mexico occurred in June 2019; 16 people became severely ill, and almost all patients had Vibrio infections, especially those involving $V$. parahaemolyticus and Vibrio albensis (CDC, 2019).
Reports of outbreaks causing life threatening infections with Vibrio species associated with the consumption of a variety of seafoods are increasing. Therefore, to understand the risk of acquiring Vibrio infections through the consumption of traditional market-based seafood, it is important to have data on the prevalence of the virulent strains in traditional marketbased seafood. This study aimed to examine the distribution of Vibrio species in sea snail (Neptunea cumingi Crosse and Busycon canaliculatu), Chinese traditional market-based seafood in the Qingdao province of China and determine the prevalence of the virulence genes of thermostable direct hemolysin $(t d h)$, thermostable direct hemolysin-related hemolysin (trh), and thermolabile hemolysin ( $t h$ ) in $V$. parahaemolyticus isolates for food safety purposes.

\section{MATERIALS AND METHODS}

\section{Sample Collection}

In total, 20 samples of sea snail were randomly collected from four traditional markets in the Chinese city of Qingdao $\left(36^{\circ} 04^{\prime} 01^{\prime \prime} \mathrm{N} \quad 120^{\circ} 22^{\prime} 58^{\prime \prime} \mathrm{E}\right)$ during March-September, 2018 (Figure 1). Approximately $1 \mathrm{~kg}$ of each sample was collected. All samples were packed in sterile plastic zipper bags, stored in an airtight box containing crushed ice, and transferred to the laboratory in $4 \mathrm{~h}$ for further processing.

\section{Sample Processing}

All samples were thawed and pre-incubated in buffered peptone water for 16-18 h. Thereafter the samples were serially diluted, cultured and the bacteria were counted according to standard microbiological methods. Briefly, $25 \mathrm{~g}$ of each seafood sample was transferred into a sterile stomacher bag under aseptic conditions and blended with $225 \mathrm{~mL}$ of sterile buffered peptone water using a homogenizer set at $200 \mathrm{rpm}$ for 15-20 min. Further, serial dilutions were made using sterile $0.1 \%$ peptone water to allow for culturing and bacterial enumeration.

\section{Microbiological Analysis}

Vibrio spp. were isolated and enumerated from all samples. The bacterial counts were determined using Vibrio spp. selective thiosulfate citrate bile salt (TCBS) agar plates. TCBS agar plates were inoculated by spreading $0.1 \mathrm{~mL}$ of the serial dilutions of samples and incubated at $37^{\circ} \mathrm{C}$ for $48 \mathrm{~h}$. TCBS plates were examined for the presence of either yellow, round, 2$3 \mathrm{~mm}$ diameter colonies (suspect: $V$. alginolyticus) or green, round, 2-3 mm diameter colonies (suspect: V. parahaemolyticus). Each isolated suspected Vibrio colony was streaked onto another TCBS agar plate and incubated overnight at $37^{\circ} \mathrm{C}$ to obtain pure colonies.

\section{Biochemical Characteristic Analysis}

VITEK $2^{\text {TM }}$ (BioMérieux, Marcy-l'Étoile, France) automated system was used according to the manufacturer's instructions to identify the Vibrio spp. The method is a combination of photoelectric technology, computer technology, and bacterial octet digital identification technology. VITEK is frequently used 


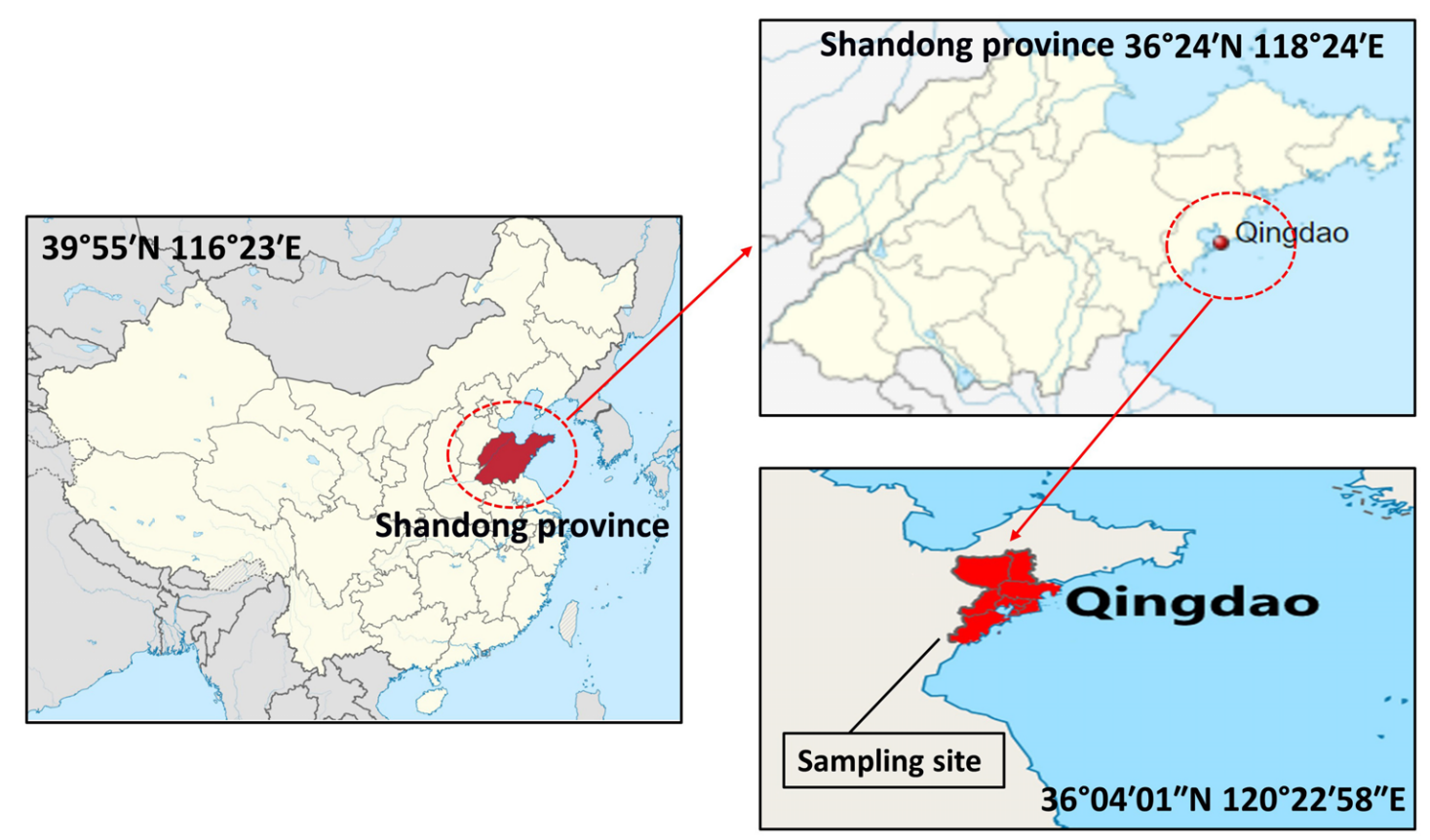

FIGURE 1 | Map of samples (sea snail) collection site in Qingdao city $\left(36^{\circ} 04^{\prime} 01^{\prime \prime} \mathrm{N} 120^{\circ} 22^{\prime} 58^{\prime \prime} \mathrm{E}\right)$ of Shandong province, China.

for identification owing to its simplicity, accuracy and prompt outcome (Funke et al., 1998). In VITEK $2^{\text {TM }}$ system, each test card contained 64 wells to carry out biochemical reactions (Funke et al., 1998). Each group was divided into three terms, and each positive reaction in the group was evaluated as 1, 2, 4, and then the values of each group were calculated. Based on the 64 biochemical reactions, biological identification numbers were obtained. With the help of the computer, the test cards were read after every $15 \mathrm{~min}$ and the dynamic reaction changes could be observed via optical scanning of the substrate of each reaction hole.

The specific operation procedure was as follows. The isolated bacteria were streaked on TCBS plates and incubated for $24 \mathrm{~h}$ at $35^{\circ} \mathrm{C}$. A homogeneous suspension was prepared with an optical density $(600 \mathrm{~nm})$ between 0.55 and 0.63 , measured with a DensiCHEK ${ }^{\mathrm{TM}}$ VITEK Caliper. Sixty-four biochemical tests were performed using the gram-negative bacteria identification VITEK cards. The VITEK biochemical tests for Vibrio parahaemolyticus (yellow color colony in TCBS medium) are described positive for indole, nitrate reduction, citrate utilization, and growth at $7 \% \mathrm{NaCl}$, while negative for urease, arginine, sucrose fermentation, swarming, and growth at $10 \% \mathrm{NaCl}$. Whereas Vibrio alginolyticus (appeared as green color colony in TCBS medium) are positive for indole, VogesProskauer (VP), nitrate reduction, citrate utilization, sucrose fermentation, swarming, and growth at 7 and $10 \% \mathrm{NaCl}$, while negative for urease, arginine.

\section{Virulence Gene Detection}

The virulence genes, namely th (450 bp) (Gutierrez West et al., 2013), tdh (251 bp) (Tada et al., 1992), and trh (250 bp) (Tada et al., 1992), were tested by polymerase chain reaction (PCR) amplification. The sequences of primers were as follows:

th-F: $5^{\prime}$-AAAGCGGATTATGCAGAAGCACTG-3'; thR: 5'-GCTACTTTCTAGCATTTTCTCTGC-3'; tdh-F: $5^{\prime}$ CCACTACCACTC TCATATGC-3';

tdh-R: $\quad 5^{\prime}$-GGTACTAAATGGCTGACATC-3'; trhF: $\quad 5^{\prime}$-GGCTCAAAATGGTTAAGCG-3'; trh-R: 5'-CATTTCCGCTCTCATATGC-3'.

The PCR was carried out in a final volume of $20 \mu \mathrm{L}$ containing $1 \times$ PCR buffer (containing $\mathrm{MgCl}_{2}$ ) (Takara, Dalian, China), $0.5 \mathrm{mM}$ dNTPs (Takara), $0.25 \mu \mathrm{M}$ forward primer and reverse primer (Songon, Shanghai, China), 1.0 U Taq polymerase (Takara), and $50 \mathrm{ng}$ genomic DNA. The PCR amplification was conducted under the following conditions: initial denaturation at $94^{\circ} \mathrm{C}$ for $5 \mathrm{~min}$, followed by 30 cycles of denaturation at $94^{\circ} \mathrm{C}$ for $1 \mathrm{~min}$, annealing at $55^{\circ} \mathrm{C}$ for $45 \mathrm{~s}$, and extension at $72^{\circ} \mathrm{C}$ for $45 \mathrm{~s}$, and an additional 7 -min extension at $72^{\circ} \mathrm{C}$. The amplicons were analyzed by $1.2 \%$ agarose gel electrophoresis and photographed in the gel imaging system (Vilber Lourmat, France). The amplicons were gel purified by TIANgel Midi Purification Kit (Tiangen) and send to Sangon Biotech Co., Ltd. for sequencing to confirm the identities. The sequence data were compared with those in the NCBI nucleotide sequence database by means of Basic Local Alignment Search Tool.

\section{RESULTS AND DISCUSSION}

Total 35 Vibrio spp. were isolated from the tested 20 seafood samples using selective (TCBS) agar medium. All the isolates 
TABLE 1 | Positive samples of Vibrio species in sea snail available in a traditional market in Qingdao city, Shandong province, China.

\begin{tabular}{|c|c|c|c|c|c|}
\hline \multirow[t]{2}{*}{ Positive sea snail samples } & \multirow[t]{2}{*}{ TCCB Agar plate* } & \multirow[t]{2}{*}{ VITEK Identification } & \multicolumn{3}{|c|}{ Presence of virulence gene } \\
\hline & & & tlh & tdh & trh \\
\hline 1 & +ve for Vibrio spp. & $V$. alginolyticus & $-v e$ & $-v e$ & $-v e$ \\
\hline 2 & +ve for Vibrio spp. & V. alginolyticus & $-v e$ & $-v e$ & $-v e$ \\
\hline \multirow[t]{2}{*}{3} & +ve for Vibrio spp. & V. alginolyticus & $-v e$ & $-v e$ & $-v e$ \\
\hline & & V. parahaemolyticus & $+v e$ & $-v e$ & +ve \\
\hline \multirow[t]{2}{*}{4} & +ve for Vibrio spp. & V. alginolyticus & $-v e$ & $-v e$ & $-v e$ \\
\hline & & V. parahaemolyticus & $+v e$ & $-v e$ & $-v e$ \\
\hline \multirow[t]{2}{*}{5} & +ve for Vibrio spp. & V. alginolyticus & $-v e$ & $-v e$ & $-v e$ \\
\hline & & V. parahaemolyticus & $+v e$ & $-v e$ & $-v e$ \\
\hline 6 & +ve for Vibrio spp. & $V$. alginolyticus & $-v e$ & - ve & $-v e$ \\
\hline \multirow[t]{2}{*}{7} & +ve for Vibrio spp. & V. alginolyticus & $-v e$ & $-v e$ & $-v e$ \\
\hline & & V. parahaemolyticus & $+v e$ & $-v e$ & $-v e$ \\
\hline \multirow[t]{3}{*}{8} & +ve for Vibrio spp. & $V$. alginolyticus & $-v e$ & $-v e$ & $-v e$ \\
\hline & & V. parahaemolyticus & $+v e$ & $-v e$ & $-v e$ \\
\hline & & V. vulgaris & $-v e$ & $-v e$ & $-v e$ \\
\hline \multirow[t]{3}{*}{9} & +ve for Vibrio spp. & $V$. alginolyticus & $-v e$ & $-v e$ & $-v e$ \\
\hline & & V. parahaemolyticus & $+v e$ & $-v e$ & $-v e$ \\
\hline & & V. vulgaris & & & \\
\hline \multirow[t]{2}{*}{10} & +ve for Vibrio spp. & V. alginolyticus & $-v e$ & $-v e$ & $-v e$ \\
\hline & & V. parahaemolyticus & +ve & $-v e$ & $-v e$ \\
\hline \multirow[t]{2}{*}{11} & +ve for Vibrio spp. & V. alginolyticus & $-v e$ & $-v e$ & $-v e$ \\
\hline & & V. parahaemolyticus & $+v e$ & $-v e$ & $+v e$ \\
\hline
\end{tabular}

${ }^{*}$ TCBS, thiosulfate citrate bile salt (Vibrio spp. Selective medium); -ve: negative; +ve: positive. Bold value is highlight of the positive result.

were tested for three major Vibrio species: V. parahaemolyticus, $V$. alginolyticus, and V. vulgaris using VITEK test kits, and tested for biochemical activity, serotype, and the presence of known virulence markers (thh, $t d h$, and trh genes). The $t d h$ and trh genes are strongly correlated with the virulence of pathogenic V. parahaemolyticus (Gutierrez West et al., 2013; Raghunath, 2015). To our knowledge, very few studies have characterized such a diverse panel of $V$. parahaemolyticus isolates in seafood samples from traditional markets at this level. Table 1 shows detailed information on the Vibrio species identified in all samples. The reliability and reproducibility of the VITEK system for Vibrio strain identification was tested every other day. Out of the 20 seafood samples, $V$. alginolyticus was identified in $55 \%(n=11)$, while $V$. parahaemolyticus was identified in $40 \%$ $(n=8)$ samples. Interestingly, V. parahaemolyticus is highly cooccurred with $V$. alginolyticus (8/11). Besides these two Vibrio spp., $V$. vulgaris was also present in $10 \%$ samples $(n=2)$, and it always co-occurred with $V$. alginolyticus and $V$. parahaemolyticus (Table 1). These results indicate that there is a high incidence of $V$. alginolyticus and $V$. parahaemolyticus in seafood distribution channels. The $V$. parahaemolyticus isolates were further tested for the $t d h$, trh, and th virulence factors. Of these, th is suggested to be an important molecular marker for the identification of V. parahaemolyticus (Gutierrez West et al., 2013). Results showed that all the VITEK-identified $V$. parahaemolyticus isolates $(n=8)$ were positive for th, thus confirming the success of the VITEK identification method. Results are good agreement with the previous published reports showing the presence of th positive $V$. parahaemolyticus in many sea food samples
(Letchumanan et al., 2014). Consistent with this Terzi Gulel and Martinez-Urtaza (2016) identified and confirmed many strains of $V$. parahaemolyticus using th virulence marker. We further noticed that among all the identified $V$. parahaemolyticus isolates, only $25.0 \%(n=2)$ were positive for trh. Moreover, all the identified $V$. parahaemolyticus isolates were $t d h$-negative. These results are consistent with the findings of Jones et al. (2012) who obtained $t d h$-negative and trh-positive isolates of Vibrio species from Canada, Maine, and Washington during the early summer, indicating a potential preferential distribution of these strains in northern areas. A study carried out by Tran et al. (2018) on 385 sea food samples demonstrated the presence of $t d h$ and trh positive $V$. parahaemolyticus strains in 25 samples. Out of theses 25 samples, only 2 samples have $V$. parahaemolyticus strains with both $t d h$ and trh gene and rest 23 samples have $V$. parahaemolyticus strains either with $t d h$ and trh virulence gene. In another similar study done by Yang et al. (2017) examined the prevalence of $V$. parahaemolyticus in various oyster, fish and shrimp samples collected form the South China domestic market and revealed 8.16 and $12.24 \% t d h$ and trh positive isolates, respectively. The difference in the distribution and evolution of $t d h$ and trh in $V$. parahaemolyticus in different samples are highly associated with geographical location, sample source and environmental conditions (Raghunath, 2015). This was also reflected in a previous report that trh-positive $V$. parahaemolyticus strains constitute a higher proportion of the total $V$. parahaemolyticus population in the Mid-Atlantic region than in other areas during the summer (DePaola et al., 2010). 
Similar to our study, $V$. alginolyticus was also found to be the most frequently isolated species in two culture-based studies from Italy and Malaysia (Elhadi et al., 2004). In 2011, an interesting study on the prevalence of $V$. parahaemolyticus in retail oysters sold at a local market in São Paulo State (Brazil) reported that $100 \%$ of the samples was contaminated with $V$. parahaemolyticus. In addition, none of the 1,943 tested isolates in that study harbored $t d h$ and/or trh gene(s) (Sobrinho et al., 2011). Our data provides the first information on exposure to Vibrio spp. in traditional market-based seafood in China. However, it must be recognized that using only culturebased methods to detect Vibrio spp. could be a limitation. In our study, the VITEK along with $t d h$, $t r h$, and $t$ th virulence factors identification system was used to identify the presence of potentially pathogenic Vibrio spp. in traditional marketbased seafood.

\section{CONCLUSION}

This study identified several Vibrio species in sea snail samples locally available in the traditional Chinese market of Qingdao city, Shandong province, China through culturing on selective media and VITEK biochemical techniques. $V$. alginolyticus was found in 55\% $(n=11)$ of selected sea snails while $V$. parahaemolyticus was found in $40 \%(n=8)$ of the samples. Besides, $V$. vulgaris was also present in $10 \%$ samples $(n=2)$, and it always co-occurred with $V$. alginolyticus and $V$.parahaemolyticus in samples. All $V$. parahaemolyticus isolates were positive for th virulence factor. In addition $2 \mathrm{~V}$. parahaemolyticus isolates found positive for trh virulence factor. Eating raw seafood in Asian countries, including China, Korea, and Japan is the main cause of emerging gastrointestinal diseases. Therefore, based on the

\section{REFERENCES}

Amagliani, G., Brandi, G., and Schiavano, G. F. (2012). Incidence and role of Salmonella in seafood safety. Food Res. Int. 45, 780-788. doi: 10.1016/j.foodres. 2011.06.022

CDC (2019). Multistate Outbreak of Gastrointestinal Illnesses Linked to Oysters Imported from Mexico. Available online at: https://www.cdc.gov/vibrio/ investigations/rawoysters-05-19/index.html (accessed June 21, 2019).

Chen, A. J., Hasan, N. A., Haley, B. J., Taviani, E., Tarnowski, M., Brohawn, K., et al. (2017). Characterization of pathogenic Vibrio parahaemolyticus from the Chesapeake Bay, Maryland. Front. Microbiol. 8:2460. doi: 10.3389/fmicb.2017. 02460

Cho, T., Kim, N., Kim, S., Song, J., and Rhee, M.-S. (2016). Survival of foodborne pathogens (Escherichia coli O157: H7, Salmonella Typhimurium, Staphylococcus aureus, Listeria monocytogenes, and Vibrio parahaemolyticus) in raw readyto-eat crab marinated in soy sauce. Int. J. Food Microbiol. 238, 50-55. doi: 10.1016/j.ijfoodmicro.2016.08.041

DePaola, A., Jones, J. L., Woods, J., Burkhardt, W., Calci, K. R., Krantz, J. A., et al. (2010). Bacterial and viral pathogens in live oysters: 2007 United States market survey. Appl. Environ. Microbiol. 76, 2754-2768. doi: 10.1128/aem.02590-09

Elhadi, N., Radu, S., Chen, C., and Nishibuchi, M. (2004). ). Prevalence of potentially pathogenic Vibrio species in the seafood marketed in Malaysia. J. Food Protect. 67, 1469-1475. doi: 10.4315/0362-028x-67.7.1469

Fujino, T., Okuno, Y., Nakada, D., Aoyoma, A., Fukai, K., Mukai, T., et al. (1953). On the bacteriological examination of Shirasu food poisoning. Med. J. Osaka Univ. 4, 299-304. results obtained in this study, further efforts are needed for rapid pathogen identification and for controlling Vibrio contamination in sea snails. Additionally, meta-analyses or systematic studies on the worldwide prevalence of antimicrobial-resistant $V$. parahaemolyticus in seafood are needed. Furthermore, to minimize the risk of $V$. parahaemolyticus infection and ensure seafood safety, consultation and collaboration between researchers and governments are urgently needed. We further suggest avoiding the consumption the raw sea snails owing to their high chance of contamination with Vibrio species.

\section{DATA AVAILABILITY STATEMENT}

The raw data supporting the conclusions of this article will be made available by the authors, without undue reservation, to any qualified researcher.

\section{AUTHOR CONTRIBUTIONS}

XSo wrote the manuscript. XSo, JZ, WY, and YW performed the experiments and analyzed the data. XSh evaluated the drafts of manuscript and approved the manuscript for submission. All authors contributed to the article and approved the submitted version.

\section{FUNDING}

This study was part of the national risk surveillance program in China.

Funke, G., Monnet, D., deBernardis, C., von Graevenitz, A., and Freney, J. (1998). Evaluation of the VITEK $2^{\mathrm{TM}}$ system for rapid identification of medically relevant gram-negative rods. J. Clin. Microbiol. 36, 1948-1952. doi: 10.1128/ jcm.36.7.1948-1952.1998

Gutierrez West, C. K., Klein, S. L., and Lovell, C. R. (2013). High frequency of virulence factor genes tdh, trh, and th in Vibrio parahaemolyticus strains isolated from a pristine estuary. Appl. Environ. Microbiol. 79, 2247-2252. doi: 10.1128/aem.03792-12

Harwood, V. J., Gandhi, J. P., and Wright, A. C. (2004). Methods for isolation and confirmation of Vibrio vulnificus from oysters and environmental sources: a review. J. Microbiol. Methods 59, 301-316. doi: 10.1016/j.mimet.2004.08.001

Heng, S.-P., Letchumanan, V., Deng, C.-Y., Ab Mutalib, N.-S., Khan, T. M., Chuah, L.-H., et al. (2017). Vibrio vulnificus: an environmental and clinical burden. Front. Microbiol. 8:997. doi: 10.3389/fmicb.2017.00997

Huehn, S., Eichhorn, C., Urmersbach, S., Breidenbach, J., Bechlars, S., Bier, N., et al. (2014). Pathogenic vibrios in environmental, seafood and clinical sources in Germany. Int. J. Med. Microbiol. 304, 842-850.

Iwamoto, M., Ayers, T., Mahon, B. E., and Swerdlow, D. L. (2010). Epidemiology of seafood associated infections in the United States. Clin. Microbiol. Rev. 23, 399-411. doi: 10.1128/cmr.00059-09

Jones, J. L., Lüdeke, C. H., Bowers, J. C., Garrett, N., Fischer, M., Parsons, M. B., et al. (2012). Biochemical, serological, and virulence characterization of clinical and oyster Vibrio parahaemolyticus isolates. J. Clin. Microbiol. 50, 2343-2352. doi: $10.1128 / \mathrm{jcm} .00196-12$

Kass, P. H., and Riemann, H. P. (2006). Foodborne Infections and Intoxications, 3rd Edn. Cambridge, MA: Academic Press, 3-26. 
Lee, L.-H., Ab Mutalib, N.-S., Law, J. W.-F., Wong, S. H., and Letchumanan, V. (2018). Discovery on antibiotic resistance Patterns of Vibrio parahaemolyticus in selangor reveals carbapenemase producing Vibrio parahaemolyticus in marine and freshwater fish. Front. Microbiol. 9:2513. doi: 10.3389/fmicb.2018. 02513

Letchumanan, V., Chan, K.-G., and Lee, L.-H. (2014). Vibrio parahaemolyticus: a review on the pathogenesis, prevalence, and advance molecular identification techniques. Front. Microbiol. 5:705. doi: 10.3389/fmicb.2014.00705

Letchumanan, V., Loo, K. Y., Law, J. W., Wong, S. H., Goh, B., Ab Mutalib, N., et al. (2019). Vibrio parahaemolyticus: the protagonist of foodborne diseases. Prog. Microbes Mol. Biol. 2:a0000029. doi: 10.36877/pmmb.a0000029

Liu, W. L., Chiu, Y. H., Chao, C. M., Hou, C. C., and Lai, C. C. (2011). Biliary tract infection caused by Vibrio fluvialis in an immunocompromised patient. Infection 39, 495-496. doi: 10.1007/s15010-011-0146-0

Mala, W., Alam, M., Angkititrakul, S., Wongwajana, S., Lulitanond, V., Huttayananont, S., et al. (2016). Serogroup, virulence, and molecular traits of Vibrio parahaemolyticus isolated from clinical and cockle sources in northeastern Thailand. Infection Genet. Evol. 39, 212-218. doi: 10.1016/j. meegid.2016.01.006

Raghunath, P. (2015). Roles of thermostable direct hemolysin (TDH) and TDHrelated hemolysin (TRH) in Vibrio parahaemolyticus. Front. Microbiol. 5:805. doi: $10.3389 /$ fmicb. 2014.00805

Sobrinho P, S. C, Destro, M. T., Franco, B. D., and Landgraf, M. (2011). Occurrence and distribution of Vibrio parahaemolyticus in retail oysters in São Paulo State, Brazil. Food Microbiol. 28, 137-140. doi: 10.1016/j.fm.2010.09.006

Su, Y. C., and Liu, C. C. (2007). Vibrio parahaemolyticus: a concern of seafood safety. Food Microbiol. 24, 549-558. doi: 10.1016/j.fm.2007.01.005

Tada, J., Ohashi, T., Nishimura, N., Shirasaki, Y., Ozaki, H., Fukushima, S., et al. (1992). Detection of the thermostable direct hemolysin gene (tdh) and the thermostable direct hemolysin-related hemolysin gene (trh) of Vibrio parahaemolyticus by polymerase chain reaction. Mol. Cell. Probes 6, 477-487. doi: 10.1016/0890-8508(92)90044-X

Terzi Gulel, G., and Martinez-Urtaza, J. (2016). Molecular characterizations of Vibrio parahaemolyticus in seafood from the Black Sea, Turkey. Lett. Appl. Microbiol. 62, 494-500. doi: 10.1111/lam.12579

Tran, T. H. T., Yanagawa, H., Nguyen, K. T., Hara-Kudo, Y., Taniguchi, T., and Hayashidani, H. (2018). Prevalence of Vibrio parahaemolyticus in seafood and water environment in the Mekong Delta, Vietnam. J. Veter. Med. Sci. 80, 1737-1742. doi: 10.1292/jvms.18-0241

Weissfeld, A. S. (2014). Infections from eating raw or undercooked seafood. Clin. Microbiol. Newslett. 36:3.

Yang, Y., Xie, J., Li, H., Tan, S., Chen, Y., and Yu, H. (2017). Prevalence, antibiotic susceptibility and diversity of Vibrio parahaemolyticus isolates in seafood from South China. Front. Microbiol. 8:2566. doi: 10.3389/fmicb.2017. 02566

Zhang, S. S., Han, L. W., Shi, Y. P., Li, X. B., Zhang, X. M., Hou, H. R., et al. (2018). Two novel multi-functional peptides from meat and visceral mass of marine sanil Neptunea arthritica cumingii and their activities in vitro and in vivo. Mar. Drugs 16:473. doi: $10.3390 / \mathrm{md} 16120473$

Conflict of Interest: The authors declare that the research was conducted in the absence of any commercial or financial relationships that could be construed as a potential conflict of interest.

Copyright (c) 2020 Song, Zang, Yu, Shi and Wu. This is an open-access article distributed under the terms of the Creative Commons Attribution License (CC BY). The use, distribution or reproduction in other forums is permitted, provided the original author(s) and the copyright owner(s) are credited and that the original publication in this journal is cited, in accordance with accepted academic practice. No use, distribution or reproduction is permitted which does not comply with these terms. 